\title{
The Role of NBEMS in Medical Education in the Times of COVID-19 Pandemic: Converting Challenge into Opportunity for Innovation!
}

\author{
Pawanindra Lal ${ }^{1}$ (D) \\ Received: 21 January 2022 / Accepted: 9 February 2022/Published online: 20 February 2022 \\ (c) Association of Surgeons of India 2022
}

The National Board of Examinations was set up in the year 1975 as the examination wing under the National Academy of Medical Sciences, under the president ship of Dr S Padmavathi, the famous cardiologist. As the body was entrusted with the conduct of postgraduate examinations and its role became more defined, it was formally incorporated as a society and was registered on the 1st of March 1982, as an independent organization working as an "autonomous body" under the Ministry of Health and Family Welfare, Government of India. Since then, the National Board of Examinations has been conducting post graduate level examinations in broad specialties and post-doctoral level examinations in the super specialities. Initially, the Diplomate of the National Board (DNB) examinations was an extra examination being taken by candidates who were qualified with MD/MS or $\mathrm{DM} / \mathrm{MCh}$ to get an extra degree of DNB in that particular speciality. In the 1980s, National Board of Examinations started recognizing training for candidates in broad speciality and super specialty in hospitals, outside the National Medical Commission (erstwhile Medical Council of India), that is outside the university hospitals, such as the non-medical college government institutions, public sector undertaking hospitals and private hospitals. Soon this role expanded in a very big way and 3-year teaching programme was extensively started in the whole country in these hospitals. Candidates were selected by a separate entrance examination conducted by NBE. They were trained in the speciality in which they were admitted, and they would pass the exit examination, and they be awarded the DNB qualification. This exit examination became the benchmark of quality and high standards in the country as the panel of examiners was drawn from the best of experts from the entire country and the candidates and the examiners had no previous interaction

Pawanindra Lal

profplal@gmail.com

1 Department of Surgery, Maulana Azad Medical College, Room No 202, BL Taneja Block, New Delhi 110002, India leading to unbiased assessment. The Government of India by notification in the Gazette dated 3.10.1994 has equated the DNB qualifications with the MD/MS qualifications for broad specialties and DM/MCh qualifications for super specialities. With this, the training and qualification become at par with those trained from the university/medical college under the NMC (erstwhile MCI). Currently, there is complete equivalence for those trained in NBE accredited institutions greater than 500 beds. However, those from less than 500 bedded hospitals are required to do an extra year of senior residency in a medical college to be considered as recognized qualification for the purpose of teaching only.

Soon the entrance examinations became more centralized and for the last 10 years or so, a common entrance examination known as the All India Post Graduate Entrance Examination got started which later got renamed as the National Eligibility cum Entrance Test (NEET-PG) for the PG entrance examination. A similar one NEET-SS for the super speciality examination is also conducted by NBE. All the admissions for the DNB/DrNB courses are being conducted through these National Post Graduate Entrance Examinations NEET-PG/NEET-SS, respectively.

Currently, the National Board of Examinations, which has now been rechristened into a new name, the National Board of Examinations in Medical Sciences (NBEMS), has under its purview, more than 800 institutions in the entire country, which are imparting training in more than 80 subjects, covering both broad and super specialties with more than 10,000 students enrolled, every year for various types of courses. Broadly, there are four types of courses that are run currently under the National Board of Examinations in Medical Sciences:

1. DNB in broad speciality, which is known as the Diplomate of the National Board. This is equivalent to MD/ MS.

2. DrNB for super specialities which is Doctorate of the National Board. This is equivalent to DM/MCh. This 
name has been changed from DNB to DrNB to differentiate it as a post-doctoral degree.

3. FNB or Fellowship of the National Board for subjects after the broad specialities for skills enhancement.

4. FNB-PD or Fellowship of the National Board PostDoctoral for similar skills enhancement after their postdoctoral degree, which is the DrNB/DM/MCh.

Currently, the admission for the DNB broad specialities is through the NEET-PG examination, and since 2021, there is a centralized counselling conducted by the Medical Counselling Committee (MCC) of the DGHS. The admission to the DrNB is through the NEET-SS examination also has a common counselling conducted by the MCC. The admission to the Fellowship of the National Board is through the Fellowship Entrance Test (FET) conducted by NBEMS.

Very interestingly, the National Board of Examinations in Medical Sciences also has a 2-year post-diploma programme for those students who have done a diploma in the broad specialities to enable them to get an opportunity to be trained further for 2 years and to be awarded with a DNB in the broad speciality. These students who are having their diploma qualifications appear in the Post Diploma Common Entrance Test (PDCET) conducted by NBEMS, and through this entrance test, they enter this 2-year DNB programme which is known as the secondary DNB. So, DNB training is comprising of 3-year courses, which are through the NEET-PG and the 2-year courses for post diploma holders through the separate entrance test conducted by the NBEMS. NBEMS conducts several DNB/DrNB/FNB/ FNB-PD Courses (www.natboard.edu.in). Of these, surgical post-doctoral Doctorate of NBEMS (DrNB) courses are highlighted in Tables 1 and 2.

The responsibility of the NBEMS is not just limited to the conduct of training in postgraduate broad speciality subjects and in post-doctoral subjects across its various hospitals in the country but also to organize the common entrance

Table 1 Post MD/MS/DNB 3-year DrNB courses

\begin{tabular}{ll}
\hline 1. & Cardiovascular and thoracic surgery \\
2. & Neurosurgery \\
3. & Paediatric surgery \\
4. & Plastic and reconstructive surgery \\
5. & Surgical gastroenterology \\
6. & Surgical oncology \\
7. & Thoracic surgery \\
8. & Urology \\
9. & Vascular surgery \\
10. & Endocrine surgery \\
11. & Hepato-pancreato-biliary surgery \\
12. & Paediatric cardio-thoracic and vascular surgery \\
\hline
\end{tabular}

Table 2 Post MBBS 6-year DrNB courses

\begin{tabular}{ll}
\hline 1. & Cardiovascular and thoracic surgery (direct 6-year course) \\
2. & Neurosurgery (direct 6-year course) \\
3. & Paediatric surgery (direct 6-year course) \\
4. & Plastic and reconstructive surgery (direct 6-year course) \\
\hline
\end{tabular}

examinations, the NEET PG examination and the NEET-SS examinations, which are the route of entry for students to the postgraduate courses and super speciality courses, respectively. This responsibility has been entrusted to the National Board of Examinations in Medical Sciences by the Ministry of Health and Family Welfare, Government of India, and has been approved by the Supreme Court of India and the National Medical Commission (erstwhile MCI).

It is easy to understand that there is a huge responsibility in the conduct of a fair uniform examination at the national level and to keep it away from any kind of controversies or complaints. The National Board of Examinations in Medical Sciences has evolved in this examination process over the years in many ways.

It started with the Optical Marking Recording (OMR) sheet-based examination, which was a pen and paper examination but over the last few years has been converted into a fully computer-based test (CBT), wherein the candidates are randomly allotted their centres on the basis of their test city of choice. The question paper is released from the NBEMS only $30 \mathrm{~min}$ before the start of the examination, after all entry to the centres is closed. Each candidate gets independent complete time as per the examination duration with individual system clocking. The questions appear on the screen of the particular computer on which the candidate is seated and the questions and the answers are all jumbled and they are able to use the computer itself to place the right or the wrong answer, or leave a particular choice, and the whole process is encrypted, several times, so that the identity of the candidate is not revealed and is deciphered only when the result is completely ready and processed by the NBEMS. These processes are carried out under our most advanced software and validated and secured by encryptions at various levels at it is these processes that have given the NBEMS that credibility that it has earned for conducting fair and transparent examinations in the entire country without any blemish of leakage of papers, etc.

Over the years, the exit examinations for the broad speciality and super speciality trainees have also set a very high level of benchmark such that even the university students with their MD/MS in the broad specialties or DM/MCh in the super speciality like to take this exit examination to add that degree in front of their name, a DNB or a DrNB as the case may be, to prove to the world that their qualification given by the university examination has been attested 
even by the highest uniform authority in the country, i.e. the National Board of Examinations in Medical Sciences. Such is the reputation of the examinations conducted by the organization, and over the years, it has grown into a very high stature due to the processes and credibility that has come with it over several years of hard work and perseverance. Interestingly, many countries outside India, including UK, recognize DNB/DrNB more easily than MD/MS degrees from the university/colleges.

\section{Introduction of OSCE-Based National Exit Speciality Exit Examinations}

The last 22 months has seen India face a pandemic of unprecedented history which affected education and examination not only in India but in the entire world, and in such crisis, conduct of exit examinations for candidates who were completing their training in broad specialties or super speciality became a very challenging issue. The National Board of Examinations in Medical Sciences created history by evolving Objective Structured Clinical Examination (OSCE)based exit examination which was spearheaded by the Executive Director of the NBEMS, and these OSCE-based exit examinations were conducted from July 2020 to November 2021 for various batches of the students, and more than 12,000 students were able to appear in these examinations with more than $80 \%$ success rate.

The OSCE-based examination involved centrally delivered OSCE for 25 stations, which were broadcast to all the centres at the same time, and these OSCE stations became actually virtual stations where instead of the candidate moving from one station to the other, the OSCE station was moving in front of their eyes in the form of a PowerPoint slide, and the candidates were expected to write the answers to the questions and the clinical situations posed in front of them in that particular station on the answer sheet. Individual answer sheets were used for each station. All these answer sheets were scanned and received at the Office of NBEMS in New Delhi on the same day.

The answer sheets were evaluated in the NBEMS according to the model answer key prepared by the examiners, so that uniform marking is possible for all students of the country. Each station was corrected by only one examiner, so that there is no discrepancy in the marking scheme as well. The second part of the clinical examination consisted of viva-voce stations that were conducted at the local examination centre with the help of local examiners present at the individual centres on face-to-face basis, and the case-based examination was conducted in the afternoon. One of the case-based cases was a virtual case sent by the NBEMS to the centre $1 \mathrm{~h}$ prior to the start of this examination, and this case was discussed by the examiners uniformly in the entire country without any disparity. The other case was a skillbased case to assess the clinical skills of the candidate with reference to the subject being examined on that particular day. Ward rounds were added in the scheme of examinations in 2021 to increase the clinical component of the examination as the COVID cases declined and non-COVID work improved in the country. With the conduct of the OSCEbased examinations, the whole process of exit examinations could be completed within a period of 2 months for the entire country for all subjects. It is a possibility that NBEMS may decide to continue a small component of the OSCE based evaluation even after the pandemic is over.

\section{Introduction of Hybrid Method of Examination and Digital Evaluation}

The conduct of theory examinations for every broad speciality or super speciality candidate has also been modified significantly in the last one year during the pandemic. The NBEMS has moved to a hybrid method of examination wherein the candidates go to the computer-based centre allotted to them. The question paper appears not in the hard paper print format but as a soft copy seen by the candidate on a computer screen or a tablet. This is released from the National Board only 30 min prior to the start of the examination like all other NEET examinations after the entry to the centre has been closed. The candidates are asked to write their answers on the answer sheets provided to them. The answer sheets are collected back and transported back to NBEMS in Delhi. Here, they are scanned, and the scanned copies are then evaluated on the computer-based terminals by the examiners appointed for various subjects in especially created Digital Evaluation Lab at two offices of the NBEMS in New Delhi, where the assessors are required to physically come and mark their copies, as seen on the screen, and also give their evaluation on the screen itself. This process has reduced the evaluation time of the theory answer sheets from 4 to 5 months to nearly 2 to 3 months now, and therefore, the whole process of examination can be finished in a more time bound manner allowing the results to be declared in a timely manner, and this has been yet another achievement and a feather in the cap of the NBEMS. This Digital Evaluation has been extended for remote evaluation of answer sheets also. The result trends of various DNB/DrNB examinations are highlighted in Figs. 1 and 2.

\section{Start of Diploma Courses by NBEMS in 2020}

Another new innovation done by the NBEMS, in the year 2020, was the start of the diploma courses under direction of the Ministry of Health and Family Welfare, Government 
Fig. 1 DrNB practical examination result pass \%
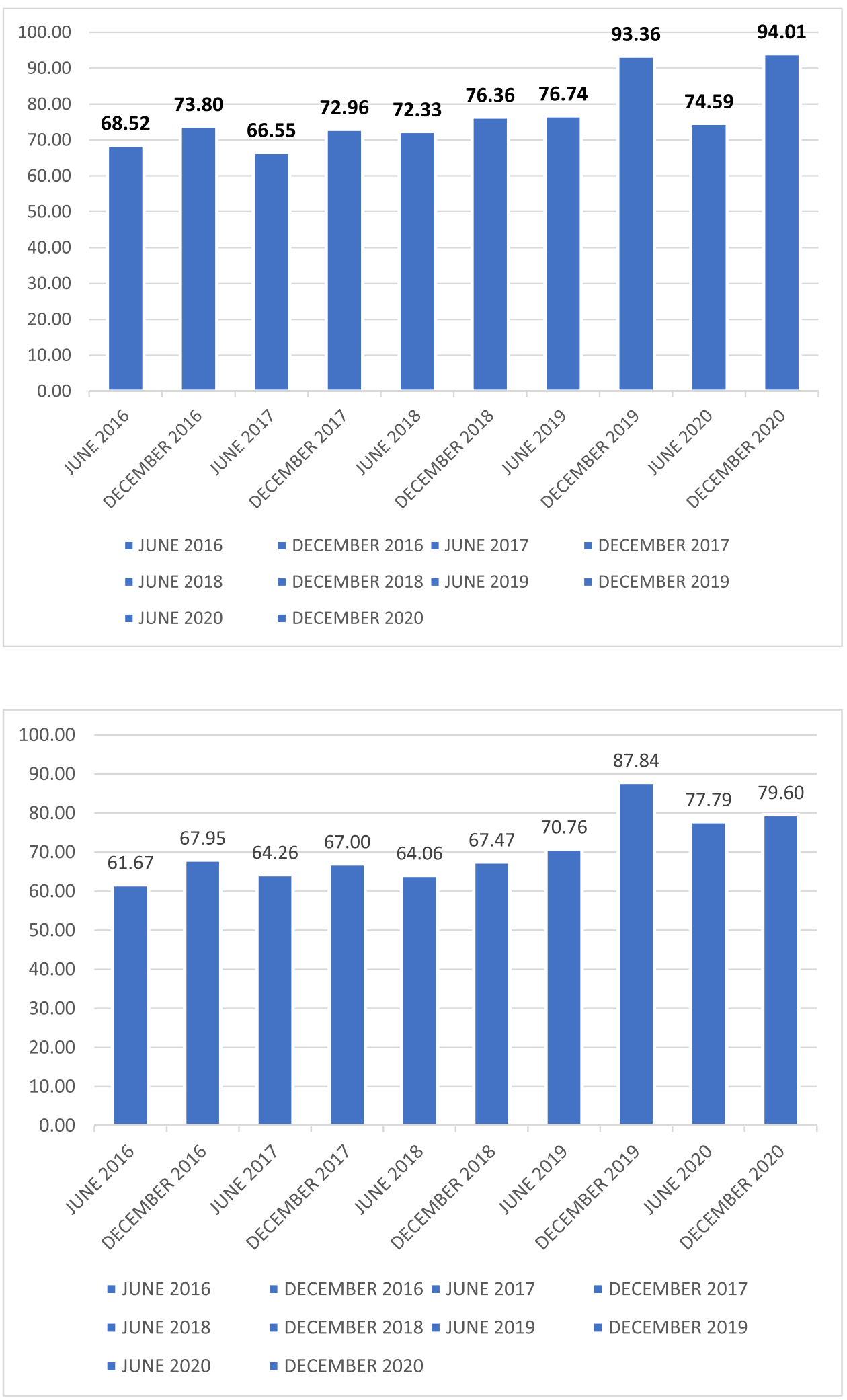

Fig. 2 DNB practical examination result pass $\%$ of India. It is important to know the history about diploma courses. We all know that 2-year diploma courses have always been going on in many broad speciality subjects in parallel and in addition to the 3-year degree courses. However, the NMC (erstwhile MCI) found that the diploma courses being done in the medical colleges leading to the 
successful post graduates with the award of diploma in the broad speciality were not being able be considered as faculty members for various medical colleges. So, a decision was taken at the highest level of the government, and it was decided that all the diploma courses to be converted into 3 -year degree courses by adding 1 more year to the course. This would lead to the generation of doctors who are degree holders, and such degree holders would then be able to be considered as faculty members in the medical colleges as only degree holders are eligible as teachers. It is well known that there is a shortage of doctors in India, and to create more doctors, more and more medical colleges are currently being established, and one of the principal requirements when medical colleges are set up is to have faculty members. So, it was not beneficial for the NMC (erstwhile $\mathrm{MCI}$ ) to have 2-year diploma courses running, and therefore, most of them have been converted into 3-year degree courses. This explanation is important because there is a false understanding by the general public and even in the medical community that the diploma courses were stopped because they were a poor programme or they were not fulfilling the purpose of the diploma course which is actually incorrect and far away from the reality. The reality was that the diploma courses have been running successfully for the past 75 years in the country and diploma holders in broad speciality have been serving the population of India in both government and private sectors to the best of their abilities and contributing to the improvement of healthcare. So, it was seen that the diploma space was currently unused and was available, and therefore, the Government of India directed the NBEMS to consider the starting of diploma courses to utilize the potential of hospitals and institutions where such training can be carried out, so that the benefit of postgraduate training can be given to the candidates. Such good diploma training programmes can result into the contribution towards healthcare of the country. Accordingly, the NBEMS got the approval mandate from the Government of India to start eight diploma programmes, and these were launched in the year 2020, the year of the pandemic, in eight subjects, i.e. anaesthesiology, obstetrics and gynaecology, paediatrics, family medicine, ophthalmology, otorhinolaryngology, tuberculosis and chest diseases and medical radiodiagnosis. These eight diplomas are in important subjects being practised in majority of the government institutions in the country even at the district hospitals and in majority of the private institutions because these are basic and essential subjects. The criteria for fulfilment for accreditation for a diploma course are much more easier for institutions to fulfil than the criteria for a degree course which are more stringent. These courses were launched primarily with the aim to target the district hospitals of India.

It is a matter of fact and well known to all of us that over the past 75 years, the current healthcare system has not been able to reach the district hospitals in the form of residency training programme, and it is also a reality that after working hours from $4 \mathrm{pm}$ in the evening to $9 \mathrm{am}$ in the morning, there are no resident doctors in any district hospital in the country. It is also well known that $60-70 \%$ of our population does depend heavily on their treatment from these district hospitals. So, this was a huge opportunity, and the NBEMS converted this challenge into an opportunity and has started the diploma courses in various district hospitals of India, and nearly 2000 seats have been added to the seat matrix of the NEET-PG entrance examination for the year 2021. This is possibly going to be the most landmark achievement with a focus on the delivery of healthcare and for the in-service doctors working in the state since independence. So 50\% of the seats will be filled by the state level doctors who are MBBS doctors who are keen to do the diploma programme, and the remaining $50 \%$ of the seats only will be filled by the NEET-PG candidates from anywhere in the country, and these are open seats. It is hoped that many more seats would be added to this bucket of diploma over the next few years, and more and more subjects will also be added to increase the scope to increase the number of seats.

The students qualifying with the diploma qualification have an in-built opportunity for career progression uniquely provided by NBEMS by way of secondary DNB programme which is a 2-year course available to all such diploma holders who can then advance themselves into a full-fledged degree of DNB. Hence, through the diploma route, 4 years will be taken to complete the DNB as compared to 3 years for a straight DNB of 3 years duration. The post diploma common entrance test (PDCET) for admission to such secondary DNB courses is conducted by the NBEMS and is taken by only a small cohort of diploma holders as per their eligible qualification for entrance into the available pool of secondary DNB seats for that year and only the subject matter of the diploma is evaluated for this entrance examination.

\section{Launch of eDoctor Logbook}

NBEMS has recently launched e-Logbook named as "eDoctorLog" in June 2021 which allows NBEMS trainees to enter their daily clinical work into the e-Logbook smartphone or computer based application available to trainees as well as their supervisors where the cases entered would be verified by their respective guides on daily basis. The centralized monitoring of the e-Logbooks would be undertaken by NBEMS. This will also allow NBEMS to get the real time update of working in various NBEMS accredited departments. This will also bring sanctity to the logbook.

Writing a thesis is essential for all DNB/DrNB candidates towards partial fulfilment of eligibility for award of DNB/ DrNB degree. Every year, thousands of DNB/DrNB trainees 
submit thesis under guidance of qualified faculty on a particular topic after collecting data and doing research. These theses are evaluated by NBEMS approved faculty member on pan India basis and accepted by NBEMS on the basis of report of these faculty. NBEMS has started receiving theses in PDF format since April 2020 through NBEMS website in addition to the hard copy and also implemented automated evaluation process of theses which has been named as "Digital Thesis Evaluation System". The theses written by DNB/ DrNB candidates and accepted by NBEMS contain a volume of research work and data which may be useful for study of medical students and learners; NBEMS has also initiated e-library of theses named as Thesis Repository System or "Shodhsagar" to preserve thesis documents through this system for the DNB/DrNB Theses accepted by NBEMS.

\section{Starting of Webinar Classes}

NBEMS in its endeavour to strengthen postgraduate training and assessment has started online webinar classes. From May 2020 NBEMS acquired a new web-based platform for launching webinar classes in all 80 specialities across the country.

With the webinar classes, up to 1000 trainees can attend a class. These webinar series are being conducted to cover seminars, panel discussions, journal clubs and case discussions with flexibility for student and faculty participation.

NBEMS is currently conducting the "National Live webinar series" for DNB/DrNB/FNB residents of NBEMS accredited institutions across the country abd has nominated NBEMS webinar coordinators in all the specialties.

Webinar classes are conducted from Monday to Saturday (7:30 AM to 8:30 PM) and have a total of 9 slots per day and
54 slots a week. In 36 specialties, national webinar coordinators have fixed the time slots, and the webinars have started on NBEMS webinar portal. NBEMS is in the process of rapidly expanding these webinars to remaining specialties as well.

\section{Conclusion}

National Board of Examinations in Medical Sciences was able to accept the COVID-19 pandemic as a challenge in a situation when the country was in a lockdown mode and convert this into an opportunity to modify and evolve the examination and evaluation system and introduce some pathbreaking innovative changes in postgraduate education. It is hoped that these reforms will strengthen delivery of optimum clinical services in the country besides improving the hands-on experience of the postgraduate students with career progression opportunities.

Acknowledgements The author would like to acknowledge the Staff of NBEMS for their support.

Author Contribution PL: Concept, plan, writing of manuscript.

\section{Declarations}

Conflict of Interest The authors declare no competing interests.

Publisher's Note Springer Nature remains neutral with regard to jurisdictional claims in published maps and institutional affiliations. 